\title{
Validity improvement of evolutionary topology optimization: procedure with element replaceable method
}

\author{
Jihong Zhu ${ }^{1 \mathrm{a}}$, Weihong Zhang ${ }^{1}$, David H. Bassir ${ }^{2}$ \\ ${ }^{1}$ The Key Laboratory of Contemporary Design \& Integrated Manufacturing Technology, Northwestern Polytechnical University \\ Xi'an, Shaanxi 710072, China \\ ${ }^{2}$ Aerospace Structures, Faculty of Aerospace Engineering, Delft University of Technology \\ Kluyverweg 1, 2629 HS Delft, The Netherlands
}

Received 20 August 2008, Accepted 7 December 2008

\begin{abstract}
The aim of this paper is to enhance the validity of existing evolutionary topology optimization procedures. As this hard-killing scheme related to the element sensitivity values may lead to incorrect predictions of inefficient elements to be removed and the value of the objective function becomes sharply deteriorated during the iterations, a check position (CP) control is proposed to prevent the erroneous topology design generated by the rejection criteria of evolutionary methods. For this purpose, we introduce a sort of orthotropic cellular microstructure (OCM) element with moderate pseudodensity that acts as a compromising element between solid element and void OCM element. In this way, all inefficient elements removed previously are automatically replaced with the moderate OCM elements depending upon the deterioration of the objective function. Erroneously removed elements are then identified in the updated finite element model through a direct sensitivity computing of the moderate OCM elements and will be finally recovered by the bi-directional element replacement. Besides, detailed structures with checkerboard patterns are eliminated by controlling the local structural bandwidth with the so-called threshold method. Typical optimization examples of structural compliance and natural frequency that were difficult to tackle are solved by the proposed design procedure. Satisfactory numerical results are obtained.
\end{abstract}

Key words: topology optimization, evolutionary method, erroneous design, check position control, moderate microstructure

\section{Introduction}

Structural optimization is always an active research topic in engineering community covering aerospace, marine and mechanical systems, etc. Nowadays, structural optimization is recognized increasingly as one of the powerful tools supporting practical structural designs. Significant achievements have been made theoretically and practically. Among others, evolutionary structural optimization (ESO) introduced by Xie and Steven [1,2] is a simple and heuristic approach for structural shape and topology design. The optimal structural layout is achieved by removing inefficient elements systematically from the ground structure on the basis of sensitivity analysis results. This approach is easily understood and implemented with less mathematical rigor. Numerical results show that a variety of problems, e.g., maximal design of structural stiffness [3], natural frequency [4,5] and buckling load [6] can be dealt with by this method. As the original ESO method of Xie and Steven was devised as a unidirectional scheme only for element removal, the restitution of the removed elements will be however unallowable during the iteration.

Further improvements were made by Querin $[7,8]$ who developed the so-called bidirectional ESO method, i.e., BESO in short. Elements can be either removed or added to the structure in each optimization step. For solid elements, the classic ESO algorithm is applied for the element removal based on the sensitivity values evaluated directly. For void regions, the process of adding elements is carried out along the boundary of the solid regions with sensitivity values approximated with the nodal displacements of the neighboring solid elements. Yang et al. $[9,10]$ applied the BESO to optimal designs of structural stiffness and natural frequency.

Both ESO and BESO have the advantage of conceptual simplicity. Tanskanen [11] explored the theoretical basis of this approach and proved that it belongs to a sequential linear programming approximate method in particular situations. Sigmund [12] believed that it is questionable to extend this approach to other cases with non-compliance objectives, multiphysics and multiple constrains. Zhou and Rozvany [13], Rozvany [14] even gave a critical view about the numerical failures of the approach. In fact, neither the stress level nor the sensitivity values can describe the element efficiency exactly. Although sensitivity value used in current ESO is said to be the change of the objective function for one element being completely removed, it is still identical to the conventional computing with infinitesimal perturbation of design variable. As one can see later, it is the unsuitable prediction of the real change of the objective function that causes numerical failures in some cases studied by Zhou and Rozvany [13].

Another common issue to be mentioned in topology optimization is how to avoid the checkerboard pattern that refers to alternations of solid and void elements over the design domain. Within this regard, the filtering scheme of Sigmund and Petersson [15] is to smooth the sensitivities of objective functions over the considered element and its eight neigh-

${ }^{a}$ Corresponding author: JH.Zhu_FEA@nwpu.edu.cn 
bors. The perimeter control method of Harber et al. [16] is to control the total interface lengths between solid and void elements. The modified version proposed by Zhang and Duysinx [17] is able to control not only checkerboard patterns but also the intermediate values of element pseudo densities. Li and Steven [18], Yang and Xie [19] also discussed sensitivity filtering scheme and perimeter control in the framework of ESO and BESO. In this work, we propose a threshold method that controls the bandwidth of solid elements to avoid the checkerboard patterns.

Element replaceable method (ERPM) proposed by Zhu and Zhang [20] recently is a variant BESO. The main difference lies in that void elements are replaced with elements of orthotropic cellular microstructures (OCM) in ERPM. The introduction of such a sort of flexible material is beneficial to prevent artificial modes [21, 22] of void areas while dynamic problems are optimized. The element deletion and growth can be interchanged between solid and void OCM elements in ERPM depending upon sensitivity results. Meanwhile, this method is also suitable to topology optimization of the structural stiffness and thermal conduction.

In this paper, a Check Position (CP) control technique is proposed and integrated with the ERPM and threshold method to prevent the deterioration of the objective function, the checkerboard pattern and the evolutionary failure due to the hard-killing guided by element sensitivity values. The concept of OCM elements with moderate pseudo-densities is introduced to verify the aberrance of the structural design so that erroneously removed elements will be recovered to repair the structure. Numerical examples will be solved to illustrate the design procedure.

\section{Limitations of Current Evolutionary Methods}

\subsection{Sensitivity analysis in ESO/BESO}

In ESO/BESO method, topology optimization problem can be basically written as

$$
\begin{aligned}
& \text { find: } \boldsymbol{x}=\left\{x_{1}, x_{2}, \ldots, x_{n}\right\} \\
& x_{i}=\left\{\begin{array}{ll}
0 & \text { void } \\
1 & \text { solid }
\end{array}(i=1,2, \ldots n)\right. \\
& \text { min } / \max f(\boldsymbol{x}) \\
& \text { s.t. } V=\sum_{i=1}^{n} V_{i} x_{i} \leq \bar{V}
\end{aligned}
$$

The design variable vector $\boldsymbol{x}$ consists of element pseudodensities. The objective function $f(\boldsymbol{x})$ is often defined by structural compliance or natural frequency calculated by FE method. The design constraint refers to the limitation of structural volume or mass.

In the framework of ESO/BESO, element deletions are carried out by virtue of the element efficiency which is measured by the sensitivity values or stress level.

For a static problem, the structural compliance defining the objective function is expressed as

$$
C=\frac{1}{2} \boldsymbol{f}^{T} \boldsymbol{u}=\frac{1}{2} \boldsymbol{u}^{T} \boldsymbol{K} \boldsymbol{u}
$$

where $\boldsymbol{f}$ and $\boldsymbol{u}$ are the vectors of loads and nodal displacements, respectively. $\boldsymbol{K}$ is the global stiffness matrix. Conven- tionally, the sensitivity value of the $i$ th element is approximated by

$$
\frac{\Delta C}{\Delta x_{i}} \approx-\frac{1}{2} \boldsymbol{u}_{i}^{T} \boldsymbol{K}_{i} \boldsymbol{u}_{i}=-C_{i}
$$

$\boldsymbol{K}_{i}$ and $\boldsymbol{u}_{i}$ are the element stiffness matrix and nodal displacement vector of the $i$ th element, respectively. $C_{i}$ is the strain energy of the $i$ th element.

For a dynamic problem, the system equation is as follows

$$
\left(\boldsymbol{K}-\omega_{(j)}^{2} \boldsymbol{M}\right) \boldsymbol{u}_{(j)}=0
$$

where $\boldsymbol{M}$ is the global mass matrix, $\omega_{(j)}$ is the $j$ th natural frequency and $\boldsymbol{u}_{(j)}$ is the corresponding modal vector. The sensitivity value is approximated by

$$
\frac{\Delta \omega_{(j)}^{2}}{\Delta x_{i}} \approx \frac{\boldsymbol{u}_{(j) i}^{T} \boldsymbol{K}_{i} \boldsymbol{u}_{(j) i}-\omega_{(j)}^{2} \boldsymbol{u}_{(j) i}^{T} \boldsymbol{M}_{i} \boldsymbol{u}_{(j) i}}{\boldsymbol{u}_{(j)}^{T} \boldsymbol{M} \boldsymbol{u}_{(j)}}
$$

$\boldsymbol{M}_{i}$ is the $i$ th element mass matrix. $u_{(j) i}$ is the $j$ th vibration shape of the $i$ th element. In both (3) and (5), $\Delta x_{i}$ is always set to be -1 indicating that element $i$ is completely removed from the design domain.

Suppose now a linear interpolation model is used for $\boldsymbol{K}_{i}$ and $\boldsymbol{M}_{i}$ as a function of $x_{i}$ with

$$
\begin{aligned}
& \boldsymbol{K}_{i}=x_{i} \boldsymbol{K}_{i 0} \\
& \boldsymbol{M}_{i}=x_{i} \boldsymbol{M}_{i 0}
\end{aligned}
$$

where $\boldsymbol{K}_{i 0}$ and $\boldsymbol{M}_{i 0}$ denote the stiffness and mass matrices of the $i$ th solid element. By differentiating (2) and (4), we can obtain the analytical sensitivities

$$
\begin{gathered}
\frac{\partial C}{\partial x_{i}}=-\frac{1}{2} \boldsymbol{u}_{i}^{T} \boldsymbol{K}_{i} \boldsymbol{u}_{i} \\
\frac{\partial \omega_{(j)}^{2}}{\partial x_{i}}=\frac{\boldsymbol{u}_{(j) i}^{T} \boldsymbol{K}_{i} \boldsymbol{u}_{(j) i}-\omega_{(j)}^{2} \boldsymbol{u}_{(j) i}^{T} \boldsymbol{M}_{i} \boldsymbol{u}_{(j) i}}{\boldsymbol{u}_{(j)}^{T} \boldsymbol{M} \boldsymbol{u}_{(j)}}
\end{gathered}
$$

In comparison, sensitivity values of (3) and (5) used in the hard-killing scheme of ESO/BESO are nothing but (7) and (8) that are unable to represent the real change of the objective function whenever the inefficient element $i$ is directly removed from the design domain. To figure out the problem, consider two kinds of sensitivities shown in Figure 1.

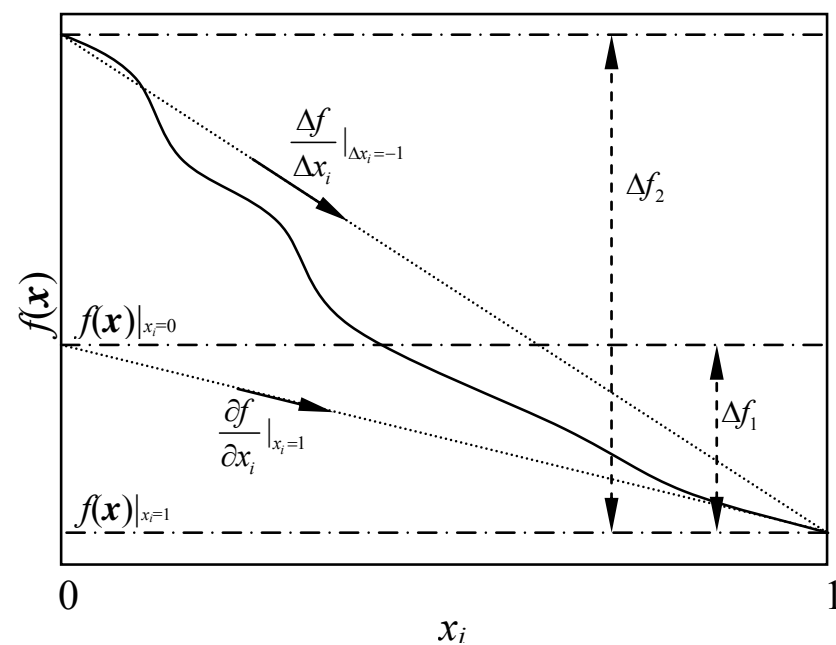

Figure 1: Objective function $f(\boldsymbol{x})$ versus the pseudo-density variable $x_{i}$ 
The solid curve in Figure 1 represents the objective function versus $x_{i}$. This is typically the case for the structural compliance with values of other terms in vector $\boldsymbol{x}$ being invariable. $\Delta f_{1}$ corresponds to the change of $f(x)$ evaluated with the analytical sensitivity value when the $i$ th element is removed by ESO/BESO from the design domain whereas $\Delta f_{2}$ represents the exact change of $f(x)$ after the removal of the $i$ th element. Clearly, both are quite different from each other.

As $\Delta f_{1}<\Delta f_{2}$, particularly when a sharp difference exists, this is the danger if related elements could be erroneously considered to be inefficient, removed and no longer recoverable during the iterations. In contrast, the change of the objective function will be overestimated when $\Delta f_{1}>\Delta f_{2}$. This is a conservative situation so that some inefficient elements are retained in the actual iteration.

Although lots of existing results have shown that the convergence of the iteration procedure is not deteriorated by $\mathrm{ESO} / \mathrm{BESO}$, the understanding of the nature of the solid curve in Figure 1 is essential to reveal the underlying trouble of ESO/BESO.

\subsection{A test example with failure of ESO}

To illustrate the phenomenon and reasons for the failure of ESO, a test example from Zhou and Rozvany [13] is analyzed here in detail. The FE model shown in Figure 2 consists of a $32 \mathrm{~m} \times 3 \mathrm{~m}$ horizontal beam and a $1 \mathrm{~m} \times 4 \mathrm{~m}$ vertical link meshed with $1 \mathrm{~m} \times 1 \mathrm{~m}$ 8-node quadratic elements. Element $A$ is located on top of the vertical link.

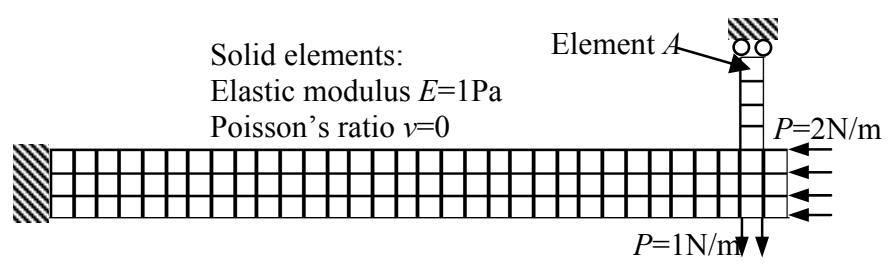

Figure 2: Test example from Zhou and Rozvany [13]

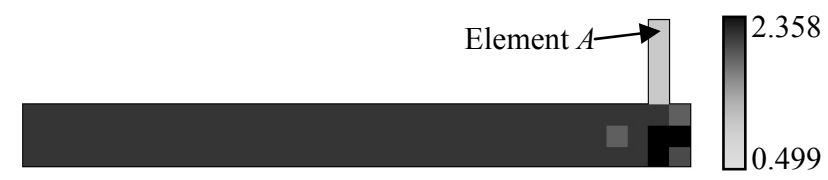

Figure 3: Distribution of the strain energy of the original structure $(\mathrm{C}=194.937 \mathrm{~J})$

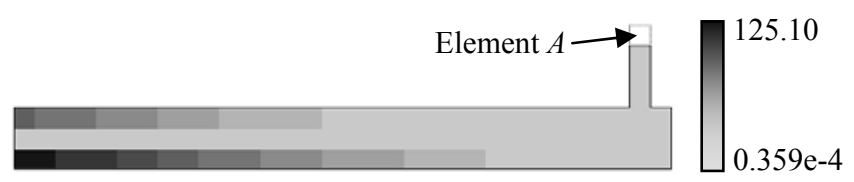

Figure 4: Distribution of the strain energy with element $A$ removed $(C=2306.88 \mathrm{~J})$

To figure out the difficulty, the initial distribution of the element strain energies which indicate the absolute sensitivity values is firstly evaluated and shown in Figure 3. It is found that element $A$ takes the lowest strain energy and should be removed in the ESO procedure. When the updated model is reanalyzed, a new distribution of the element strain energies is shown in Figure 4 with a sudden augmentation of the structural compliance $(C)$ to more than 10 times. This implies that a fatal iteration is generated. Unfortunately, the elements with the maximum strain energies are now relocated at the left bottom corner of the horizontal part so that the erroneously removed element $\mathrm{A}$ is no longer recovered by BESO.

To find out the reason for this sudden variation of the objective function, the strain energy of the total structure $(C)$ is drafted versus the variation of the elastic modulus of element $A\left(E_{A}\right)$ from $1 \mathrm{~Pa}$ to $10^{-7} \mathrm{~Pa}$ in Figure 5 where the axis of $E_{A}$ is logarithmic. A significant change of $C$ can be observed for values of $E_{A}$ between $10^{-2} \mathrm{~Pa}$ to $10^{-5} \mathrm{~Pa}$. However, the slope of the strain energy is relatively a tiny value here for element $A$ with $E_{A}$ near 1Pa so that the sharp increase of the strain energy is ignored in ESO. Thus, element A is apparently considered to be inefficient and completely removed. This is the fatal error leading to the design failure.

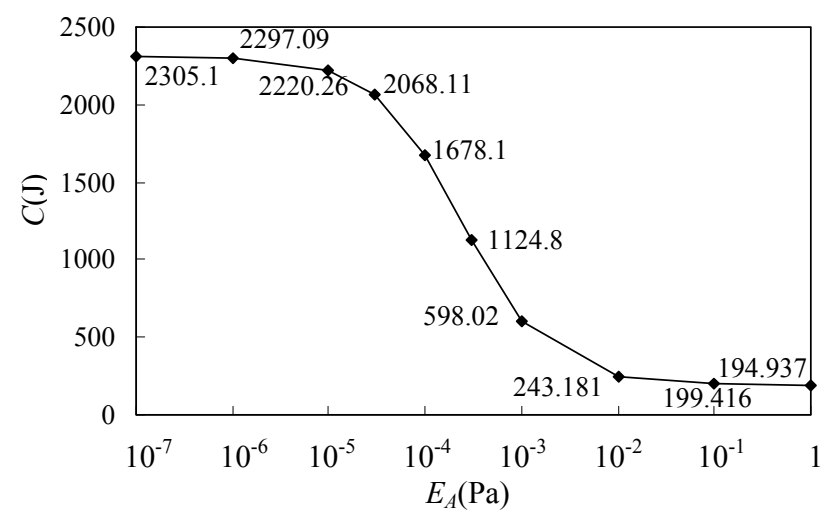

Figure 5: Global strain energy versus elastic modulus of element $A$

Based on the above discussions, the common features of questionable elements are summarized below.

1) These elements are too stiff for the actual requirement of the structure. It can be seen in Figure 5 that the structural compliance hardly changes when $E_{\mathrm{A}}$ varies between 0.01 and 1. Strain energies of these solid elements are always very low. Otherwise they won't be removed by evolutionary methods. From this point of view, if the solid-void pattern is not absolutely insisted in topology optimization, these elements may be modified to be lighter and less stiff than solid ones. Similar problems in dynamics have been discussed by Wang et al. [25]. Although it is not concerned with topology optimization, it was found that a properly positioned intermediate elastic support can raise the natural frequency of a beam to its upper limit.

2) These elements must be the main part for load-carrying, otherwise the structural compliance should not be increased so significantly after their removal. Although these elements are allowed to be less stiff, they are definitely not allowed to be completely removed. Therefore, load-carrying capacities of these elements need to be ensured.

3) When these elements are removed (or compliant enough to be considered as removed), a qualitative change of the global structural topology and the force transfer path will occur. 


\section{Ameliorations of evolutionary optimization procedures}

\subsection{New improvements in ERPM}

According to the above discussions, the traditional ESO/BESO approach cannot inspect the sharp increase of the strain energy that corresponds to high absolute sensitivity values (slope) when $E_{A}$ varies between $10^{-2} \mathrm{~Pa}$ to $10^{-5} \mathrm{~Pa}$ in Figure 5. This means that element $A$ takes the maximum strain energy of all the elements for a wide range of $E_{A}$. To understand well, the variation of strain energy of element $A$ $\left(C_{A}\right)$ is shown in Figure 6 for its elastic modulus varying from $1 \mathrm{~Pa}$ to $10^{-7} \mathrm{~Pa}$. Note that values with * refer to the maximum element strain energy of the current structure.

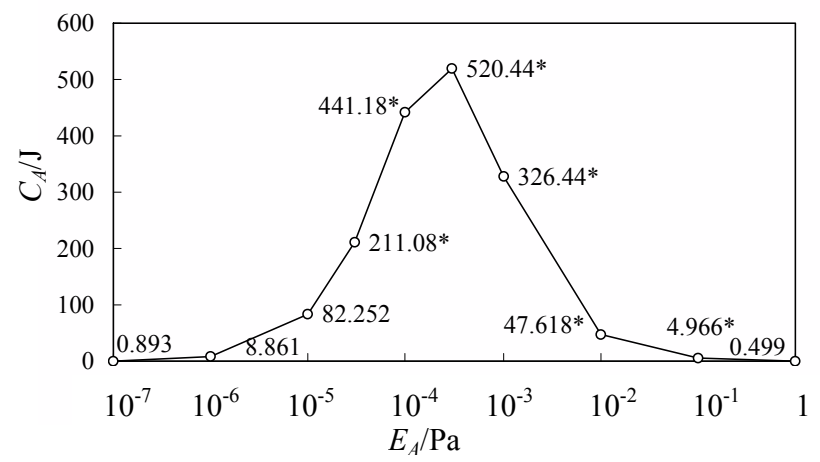

Figure 6: Strain energy of element $A$ versus its elastic modulus

By definition, $C_{A}$ is expressed as

$$
C_{A}=\frac{1}{2} \boldsymbol{f}_{A}^{T} \boldsymbol{u}_{A}=\frac{1}{2} \boldsymbol{u}_{A}^{T} \boldsymbol{K}_{A} \boldsymbol{u}_{A}=\frac{x_{A}}{2} \boldsymbol{u}_{A}^{T} \boldsymbol{K}_{A 0} \boldsymbol{u}_{A}
$$

where element model $E_{A}=x_{A} E_{0}$ is assumed with $E_{0}$ being the original elastic modulus. $\boldsymbol{f}_{A}$ and $\boldsymbol{u}_{A}$ are the element nodal force and displacement vectors, respectively. $\boldsymbol{K}_{A 0}$ is the original stiffness matrix of solid element $A$.

According to the second term in the summary of section 2.2, when $x_{A}$ varies from 1 to $10^{-2}$, element $A$ is stiff enough to be the main part carrying the load. And the current nodal force of element $A$ can be considered to be almost invariable with

$$
\boldsymbol{f}_{A}=\boldsymbol{K}_{A} \boldsymbol{u}_{A}=x_{A} \boldsymbol{K}_{A 0} \boldsymbol{u}_{A} \approx \boldsymbol{f}_{A 0}=\boldsymbol{K}_{A 0} \boldsymbol{u}_{A 0}
$$

where $\boldsymbol{f}_{A 0}$ and $\boldsymbol{u}_{A 0}$ are the original nodal force and displacement vectors, respectively. From (10), it follows that

$$
\boldsymbol{u}_{A} \approx \frac{\boldsymbol{u}_{A 0}}{x_{A}}
$$

The strain energy of element $A$ can be thus rewritten as

$$
C_{A}=\frac{x_{A}}{2} \boldsymbol{u}_{A}^{T} \boldsymbol{K}_{A 0} \boldsymbol{u}_{A} \approx \frac{1}{2 x_{A}} \boldsymbol{u}_{A 0}^{T} \boldsymbol{K}_{A 0} \boldsymbol{u}_{A 0}=\frac{1}{x_{A}} C_{A 0}
$$

An inverse proportion signifies that the right part of the curve around $x_{A}=1$ in Figure 6 is approximately a hyperbola with a tendency of decreasing from left to right. When $x_{A}$ decreases from 1 to $10^{-2}$, the strain energy of this element will increase to almost 100 times and become the maximum value of all the void and solid elements. When $x_{A}$ takes the value less than $10^{-5}$, element $A$ has nearly no contribution to the strain energy of the global structure and can be deleted. In this case, $\boldsymbol{u}_{A}$ is approximately invariable with

$$
\boldsymbol{u}_{A} \approx \boldsymbol{u}_{A R}
$$

$\boldsymbol{u}_{A R}$ is the assumptive nodal displacement vector of the removed element $A$ calculated at the common nodes of the neighboring elements. The combination of (9) and (13) gives rise to

$$
C_{A}=\frac{x_{A}}{2} \boldsymbol{u}_{A}^{T} \boldsymbol{K}_{A 0} \boldsymbol{u}_{A} \approx \frac{x_{A}}{2} \boldsymbol{u}_{A R}^{T} \boldsymbol{K}_{A 0} \boldsymbol{u}_{A R}
$$

where $\boldsymbol{u}_{A R}^{T} \boldsymbol{K}_{A 0} \boldsymbol{u}_{A R}$ is design independent. The strain energy of the element $A$ is approximated as a directly proportional function of $x_{A}$, that increases from left to right. Since $C_{A}$ is a continuous non-monotonous function of $x_{A}$, the peak of the strain energy exists when $x_{A}$ takes the value in the range of $10^{-5}$ to $10^{-2}$.

Here, the developed CP control is to remedy the erroneous element deletion. The key idea of CP is to introduce a moderate OCM element with the elastic modulus set to $0.01 \%$ $0.1 \%$ of the initial value, i.e., $10^{-4}-10^{-3} \mathrm{~Pa}$ in this problem. This element is able to capture the sharp variation of the objective function. Compared to solid elements, moderate elements of OCM microstructures will act as compromising elements between void OCM elements and solid elements but they are not compliant enough to be treated as removed ones. This provides the possibility to pick out the elements that were erroneously removed and to recover them in the design procedure. Here, void and moderate OCM models with their material properties are presented in Table 1.

Table 1: Effective material properties of OCM

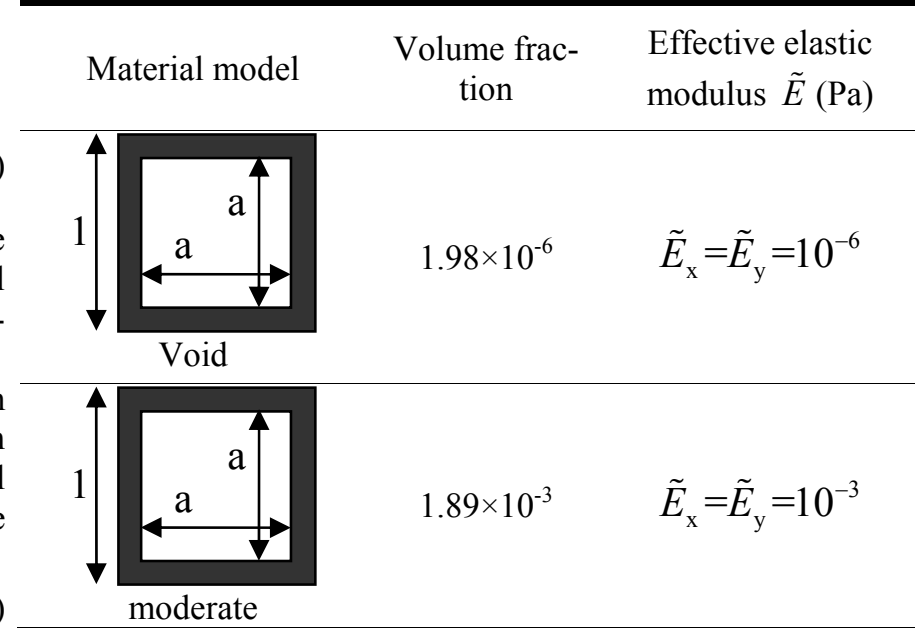

In the test example, element $A$ is now replaced with the moderate OCM element of equivalent elastic modulus $10^{-}$ ${ }^{3} \mathrm{~Pa}$. The corresponding distribution of the element strain energy is shown in Figure 7. It is seen that element $A$ substituted with the moderate OCM element takes not only a high value but also the maximum value of the element strain energies.

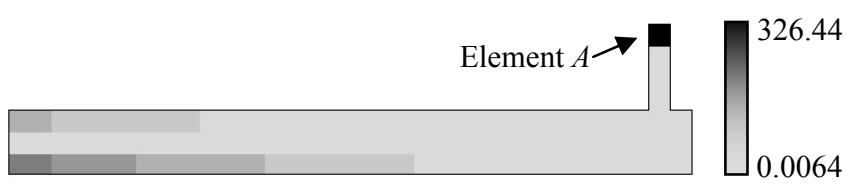

Figure 7: Distribution of the strain energy with OCM moderate element $\left(E_{A}=10^{-3} \mathrm{~Pa}, C=243.181 \mathrm{~J}\right)$ 
In this paper, the pseudo-density variable related to the moderate OCM element is assumed to be $10^{-3}$. The procedure that integrates the CP control and ERPM is as follows:

1) Calculate the design sensitivities and replace the inefficient elements with void OCM elements.

2) If a sharp degradation of the objective function is inspected against a specified threshold, e.g. the structural compliance increases to more than 5 times or the natural frequency decreases to less than $1 / 5$, the elements replaced by void OCM elements will be further replaced with the moderate OCM elements. Otherwise, a normal ERPM procedure of element removal (replaced with void OCM elements) and growth (replaced with solid elements) is carried out.

3) Based on the FE reanalysis of the updated model, moderate OCM elements having the maximum strain energies will be recovered and marked as non-designable in the next few iterations, while the other moderate elements will be replaced with void OCM elements.

To show the effect, the proposed design procedure is applied to the problem shown in Figure 2. A refined mesh shown in Figure 8 is used to generate a reasonable design.

The FE analysis is firstly carried out to compute sensitivity values and Figure 9 shows the distribution of the element strain energy. Then, all the elements with the lowest strain energy will be replaced with the void OCM elements. For such a modified model, the distribution of the element strain energies is shown in Figure 10 where the dotted line indicates the replaced area.

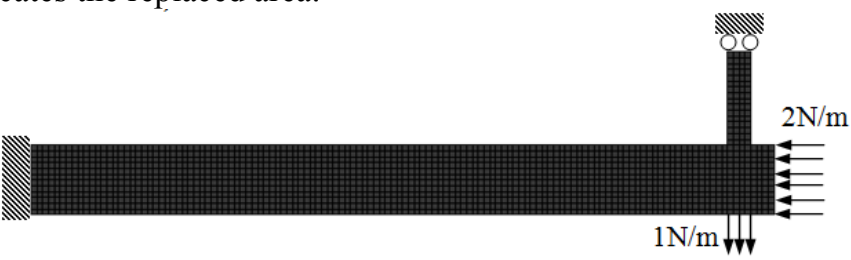

Figure 8: Refined FE model to test the improved design procedure

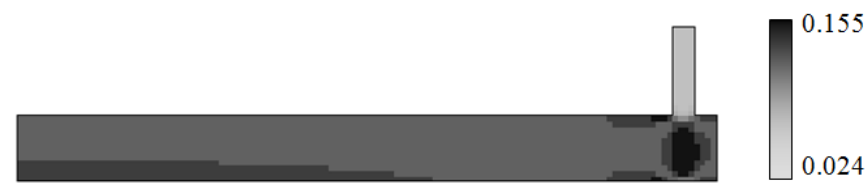

Figure 9: Distribution of the original element strain energy $(C=195.04 \mathrm{~J})$

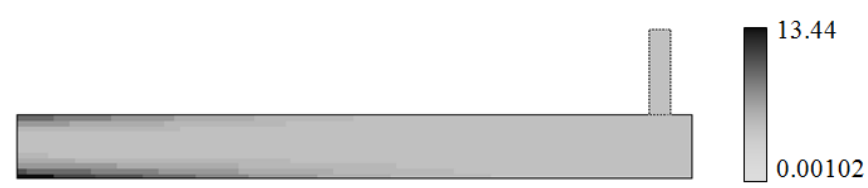

Figure 10: Distribution of the element strain energy with void OCM elements $(C=2296.64 \mathrm{~J})$

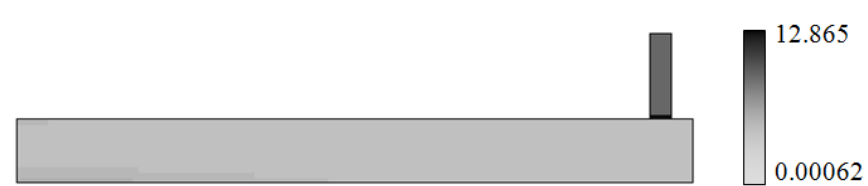

Figure 11: Distribution of the strain energy with moderate OCM elements $(C=1238.36 \mathrm{~J})$

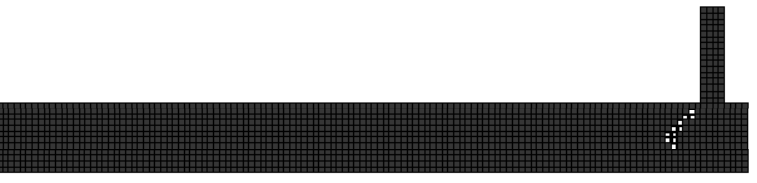

Figure 12: Structural topology with CP control after the first iteration $(C=205.485 \mathrm{~J})$

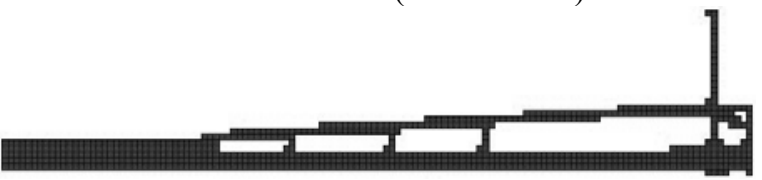

Figure 13: Final topology design with ERPM $(C=509.829 \mathrm{~J})$

The CP control is required due to the sharp augmentation from $C=195.04 \mathrm{~J}$ to $C=2296.64 \mathrm{~J}$. All the void OCM elements will be replaced with the moderate OCM elements, whose equivalent elastic modulus is $10^{-3} \mathrm{~Pa}$, i.e., $0.1 \%$ of the initial value. The maximum strain energy is redistributed over the entire structure as in Figure 11. Erroneously removed elements in the previous iteration now take the maximum values. Thus, they will be recovered with solid elements and marked as non-designable ones in next five iterations. The structural topology is shown in Figure 12 after the first iteration.

In this way, the iteration continues with the help of ERPM. The final solution is shown in Figure 13 with an amount of $40 \%$ of the total material.

The iteration histories of the objective function and volume constraint are shown in Figure 14. The diagram in Figure 14(a) shows the iteration history of the first few iterations and the effect of the CP control upon the peak augmentation of the objective function. Although elements removed erroneously are recovered and marked as non-replaceable ones in the next five steps, the objective function still jumps to very high values and will not stop popping up until iteration 44 . The convergence attains when the vertical link and the horizontal beam have almost a uniform distribution of strain energies. Figure 14(b) gives a global view of the iteration history with ignorance of objective sharp jumps in the curve. Note that the horizontal line at the volume fraction of 0.4 indicates the upper bound of the volume constraint.

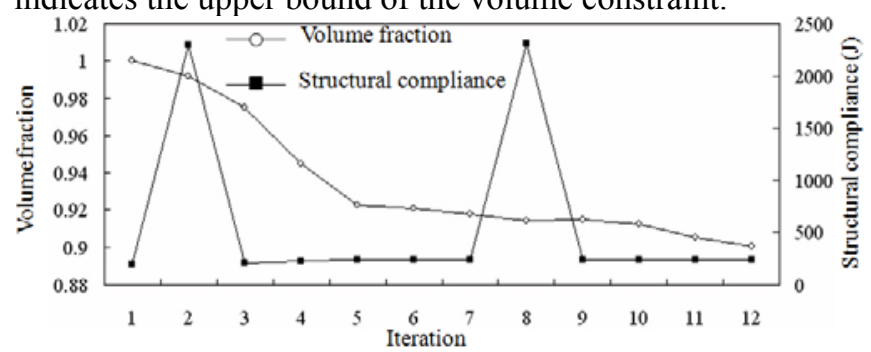

(a) The first few steps including objective jumps

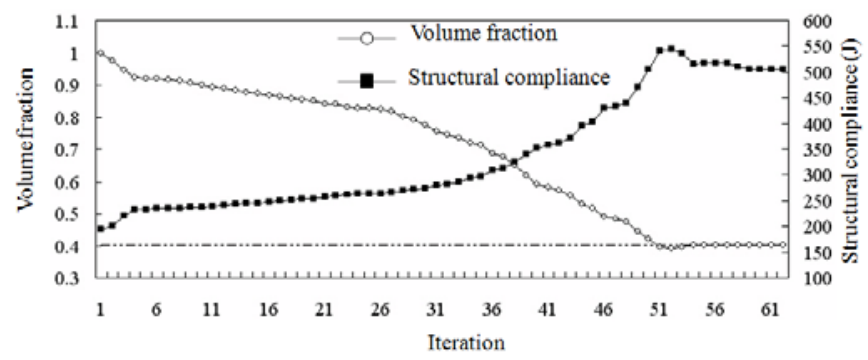

(b) The entire iteration history excluding objective jumps Figure 14: Iteration history of the tested example 


\subsection{Control of checkerboards and detailed structures}

Compared to the traditional checkerboard control method, e.g. filtering of sensitivities and perimeter constraints, the threshold method is proposed here particularly to adapt the evolutionary method like ERPM. As shown in Figure 15, both checkerboards and detailed structures can be considered as a kind of highly local alternations of solid-void elements.

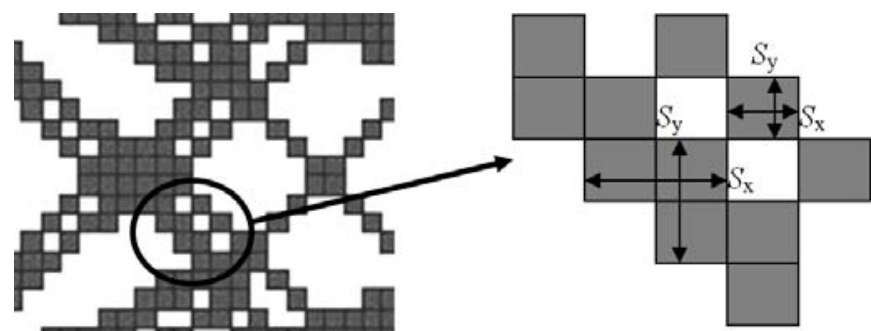

Figure 15: Identification of the checkerboards and the detailed structure

In brief, such a control procedure consists of three basic steps.

1) Threshold setup. Sensitivity values are firstly calculated by FE analysis. The threshold is then determined as the average value of all the solid elements.

2) Identification. If the local structural bandwidths $\left(S_{\mathrm{x}}\right.$ and $\left.S_{\mathrm{y}}\right)$ are less than the prescribed bandwidths $\left(S_{\mathrm{px}}\right.$ and $\left.S_{\mathrm{py}}\right)$ simultaneously, these alternating solid and void elements are identified as a detailed structure as shown in Figure 15. In particular, when $S_{\mathrm{x}}=S_{\mathrm{y}}=1$, the corresponding local structure is identified as checkerboard.

3) Control. Take a compliance design example. If the average sensitivity of the elements in the detailed structure is less than the threshold defined above, all void OCM elements surrounding solid elements of the detailed structure will be filled with solid elements to make its bandwidth reach $S_{\mathrm{px}}$ and $S_{\mathrm{py}}$. Otherwise, all solid elements in the detailed structure will be replaced with void OCM elements.

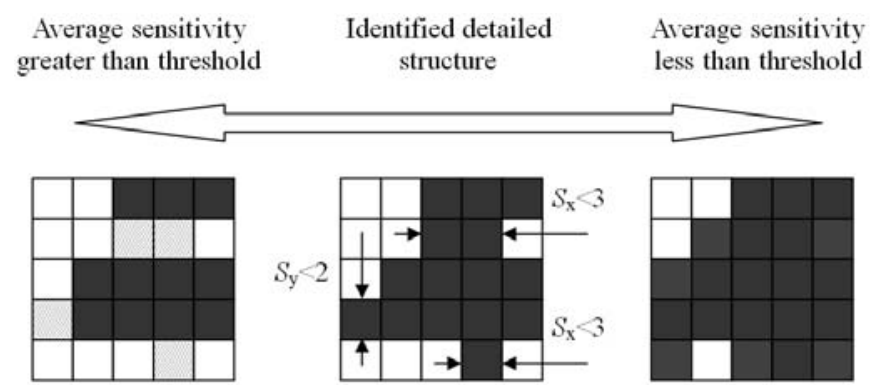

(a) Detailed structures
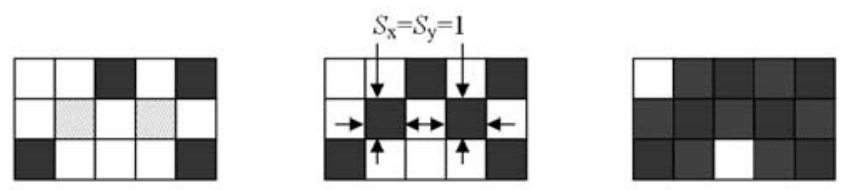

(b) Checkerboards

Solid elements

Void elements

Filled elements

Removed elements

Figure 16: Control of detailed structures and checkerboards with threshold method
The procedure is illustrated in Figure 16. When the threshold values are set to be $S_{\mathrm{px}}=3$ and $S_{\mathrm{py}}=2$, the detailed structures with $S_{\mathrm{x}}<3$ or $S_{\mathrm{y}}<2$ are then identified as shown in Figure 16(a). When the checkerboards are only controlled, the local structures with $S_{\mathrm{x}}=S_{\mathrm{y}}=1$ will be identified as shown in Figure 16(b).

To highlight the effect of the threshold method, an illustrative structure meshed with $40 \times 80$ quadrangular elements is shown in Figure 17. It is clamped at the left end and a vertical force is applied at the right end. Structure compliance is minimized and $50 \%$ of the total volume is allowed.

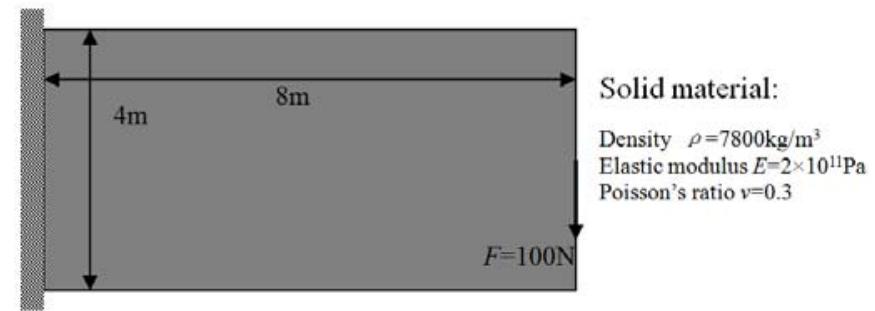

Figure 17: An illustrative optimization problem

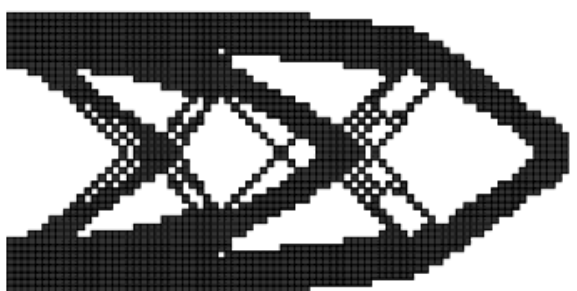

(a) Without checkerboard control

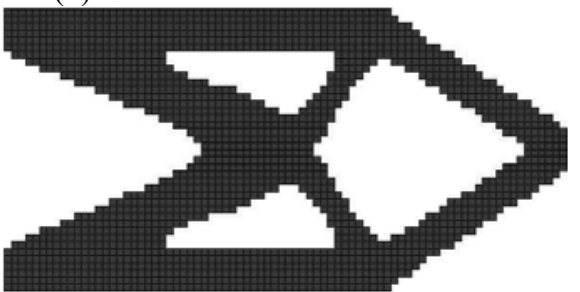

(b) Using the threshold method with $S_{\mathrm{px}}=S_{\mathrm{py}}=3$

Figure 18: Optimal topology of the illustrative example

By means of the ERPM method, two solution patterns are shown in Figure 18. One is obtained without any checkerboard control and the other is performed by using the threshold method with $S_{\mathrm{px}}=S_{\mathrm{py}}=3$.

In Figure 18(b), both the checkerboards and the detailed structures are removed in the final design. A clear topology is generated when the threshold method takes effect. The iteration history is shown in Figure 19. The convergence is achieved after about 60 iterations.

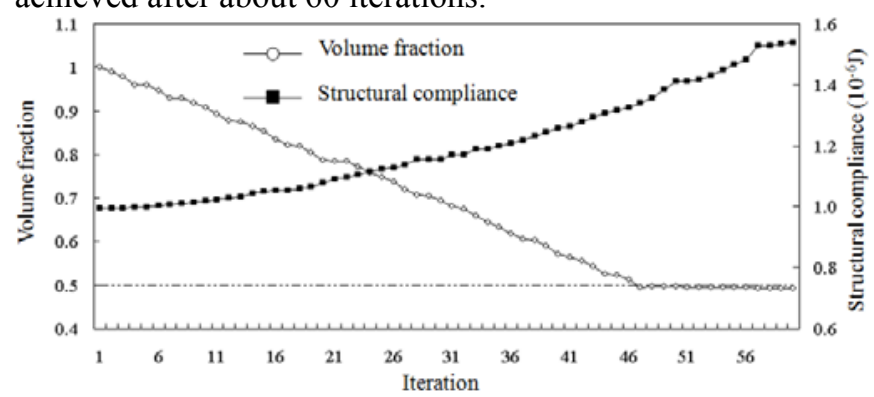

(a) Without checkerboard control 


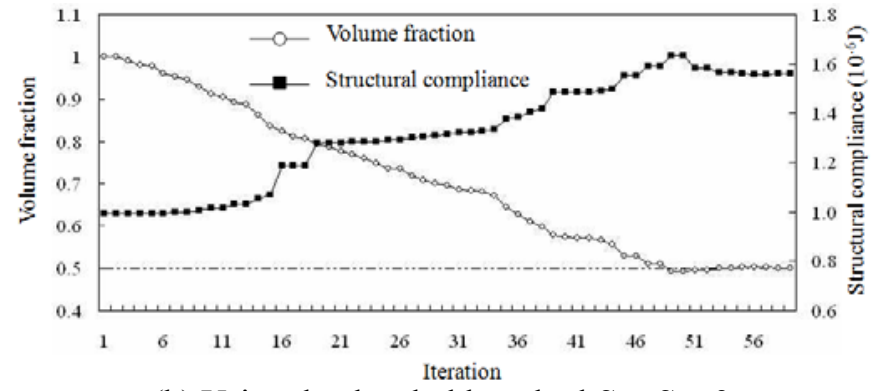

(b) Using the threshold method $S_{\mathrm{px}}=S_{\mathrm{py}}=3$

Figure 19: Iteration history of the objective function and the volume constraint

\subsection{The whole design flowchart}

In summary, the integrated ERPM design procedure is as follows:

1) Define the FE model of the entire structure and specify the design domain.

2) Carry out sensitivity analysis.

3) If it is not the first iteration and the value of the objective function is deteriorated significantly during the iteration, issue the $\mathrm{CP}$ control automatically.

4) Issue the control of detailed structures with threshold method.

5) If the limit of the material volume is not satisfied, replace the most inefficient solid elements with void OCM elements. If the limit is reached and the iteration doesn't converge, the most efficient void OCM elements will be replaced with solid elements. In stiffness design, the most efficient elements refer to those with the lowest negative sensitivity values, i.e., maximum strain energies. Whereas in the natural frequency maximization problems, the most efficient elements refer to those with the highest sensitivity values.

6) Repeat step 2) to 5) until the volume constraint is satisfied and the iteration converges.

The flowchart is described in Figure 20.

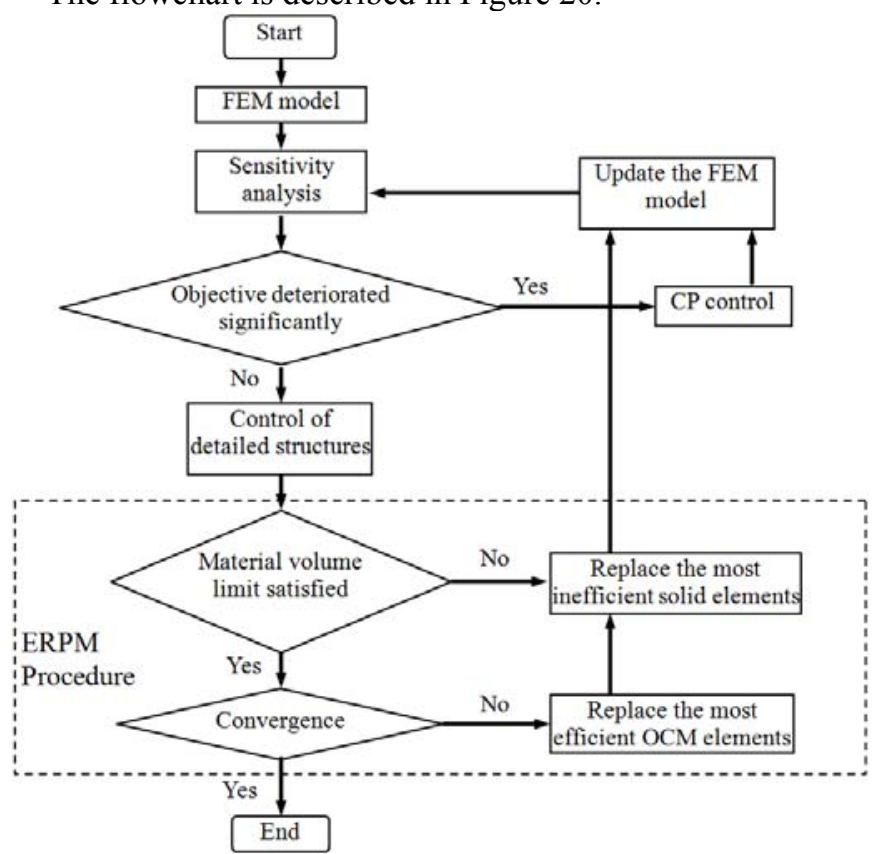

Figure 20: Flow-chart of the ERPM method

\section{Numerical examples}

In this section, two examples of maximum designs of structural stiffness and natural frequency are presented as benchmarks to give a further illustration of the evolutionary design procedure consisting of ERPM, CP control and the threshold method.

\subsection{L-shaped beam}

The problem of L-shaped beam shown in Figure 21 is proposed here. It is built up with two rectangles meshed with $20 \times 200$ and $20 \times 10$ quadratic elements, respectively. A vertical force is applied at the left bottom corner and horizontal forces are applied at all the nodes on the right end. Assume the structural compliance is minimized. The material volume is limited to $55 \%$. Threshold method corresponds to the setup of $S_{\mathrm{px}}=3$ and $S_{\mathrm{py}}=2$.

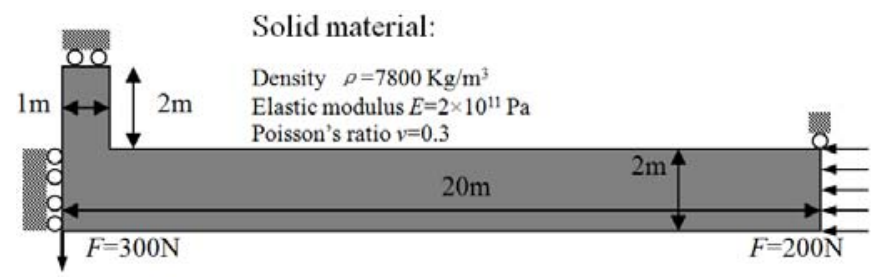

Figure 21: Design domain of the L-shaped beam

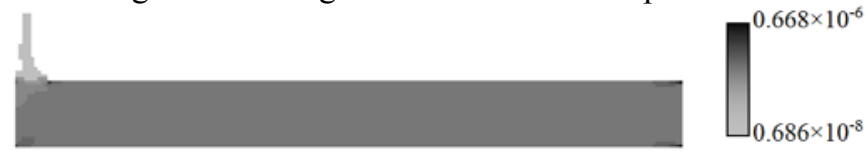

Figure 22: Distribution of strain energies at the $2^{\text {nd }}$ iteration $\left(C=0.44 \times 10^{-3} \mathrm{~J}\right)$

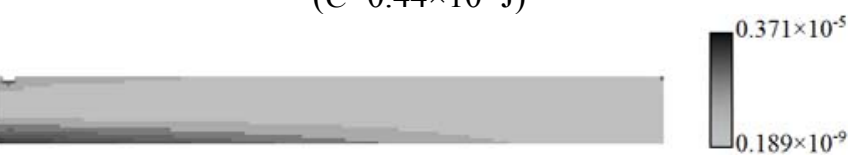

Figure 23: Distribution of the strain energy generated by the erroneous iteration $\left(C=1.35 \times 10^{-2} \mathrm{~J}\right)$

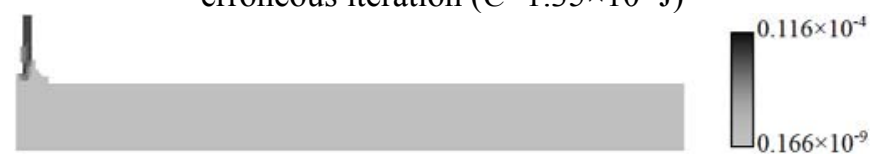

Figure 24: Distribution of the strain energy with moderate OCM elements $\left(C=1.04 \times 10^{-3} \mathrm{~J}\right)$

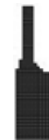

Figure 25: Structural topology generated with $\mathrm{CP}$ control $\left(C=0.44 \times 10^{-3} \mathrm{~J}\right)$
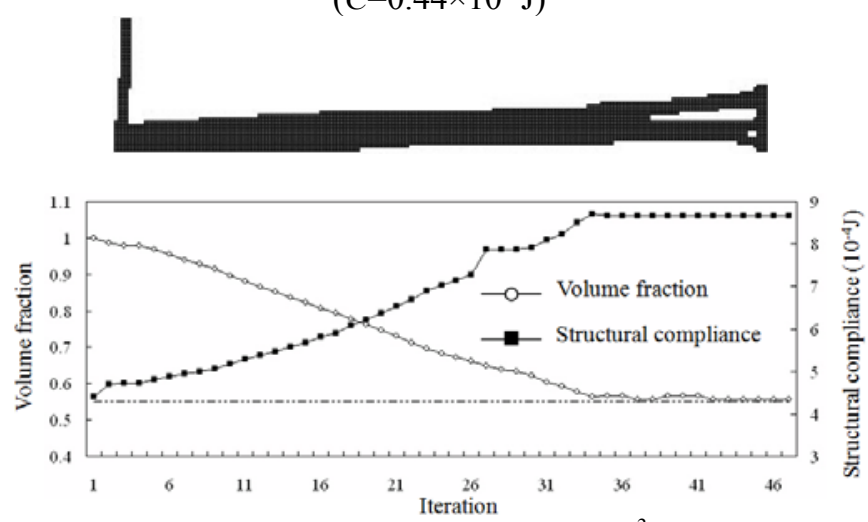

Figure 26: Final optimal design $\left(C=0.86 \times 10^{-3} \mathrm{~J}\right)$ and the iteration history 
During the iteration, several sharp increases of the structural compliance that break down the structural connectivity are figured out by the CP control. The first one occurring at the $3^{\text {rd }}$ iteration is presented here to shown the process of $\mathrm{CP}$ control. Start from the structural topology generated by the 2nd iteration, the distribution of the element strain energy is shown in Figure 22.

By removing the inefficient elements with the lowest strain energy, the new distribution of the strain energy is shown in Figure 23. This is an undesired design as the compliance increases to more than 30 times. The CP control takes the effect of replacing erroneously removed elements with moderate OCM ones whose equivalent elastic modulus is $0.1 \%$ of the original value, i.e. $2 \times 10^{-8} \mathrm{~Pa}$. The distribution of the strain energy of the updated model is shown in Figure 24.

The OCM moderate elements having the highest strain energies will be recovered as solid elements and marked as non-designable in next five steps while the others are removed again. The structural topology generated in the $3^{\text {rd }}$ iteration is shown in Figure 25.

To have a global view, the final design and the iteration history are shown in Figure 26 with all the objective jumps being removed from the curve. Values of the objective function will stop jumping in the $32^{\text {nd }}$ iteration.

\subsection{Dynamic design of a bi-clamped beam}

We propose a new test concerned with a beam supported at both ends. It is meshed with $20 \times 120$ quadratic elements. The non-designable area consists of $2 \times 20$ elements at the center as shown in Figure 27. $S_{\mathrm{px}}=3$ and $S_{\mathrm{py}}=2$ are assumed in the threshold method. The $1^{\text {st }}$ natural frequency will be maximized while only $50 \%$ of the total material is allowed.

During the design iteration, several jumps of the objective function are detected and prevented by the CP control as well. The first one occurs at the $4^{\text {th }}$ iteration. The distribution of the sensitivity values associated with the structural topology generated by the $3^{\text {rd }}$ iteration is shown in Figure 28.

Since it is a maximization problem, the updated model is obtained by removing the elements with the lowest frequency sensitivities. 12 elements are removed in this step as shown in Figure 29. It is an erroneous structure as the connectivity between the non-designable area and the structural boundary is broken.

For a dynamic problem, it is necessary to mention that sensitivity values are not always negative so that the variation of the objective function is non-monotonous and quite different from the compliance design. To show the difference, assume that the removed elements in the current iteration have a common pseudo-density value of $x_{\mathrm{R}}$. The curve of the $1^{\text {st }}$ natural frequency $f_{1}$ is plotted versus $x_{\mathrm{R}}$ in Figure 30 . When $x_{\mathrm{R}}$ decreases from $1, f_{1}$ has a short increase tendency. After that, a sharp frequency decrease takes place when $x_{R}$ diminishes from $10^{-2}$ to $10^{-5}$. This fact shows that the CP control is also required in dynamic problems.

By replacing all the erroneously removed elements with the moderate OCM elements, the newly updated model is analyzed and the distribution of the sensitivity values is shown in Figure 31. We can see that maximum sensitivity values take places for some removed elements that will be recovered while the others will be removed definitely.
Figure 32 shows the structural layout generated in this iteration with the CP control. During the whole iteration history, sudden jumps of the objective function will not stop until the $46^{\text {th }}$ iteration. The final design and the iteration history are given in Figure 33 with the removal of all jumps for the objective function.

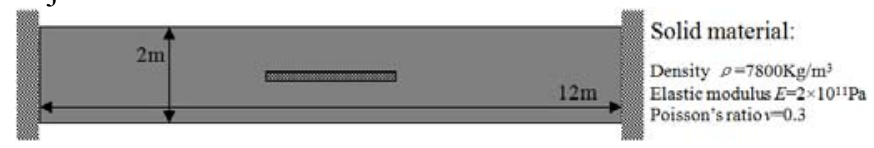

Figure 27: Design domain of the beam

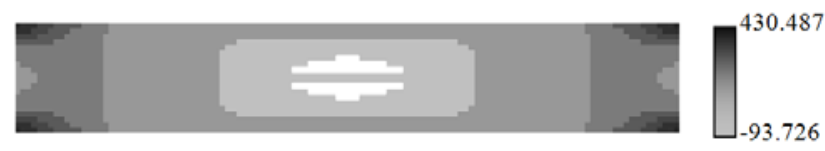

Figure 28: Sensitivity values associated with the topology generated by the $3^{\text {rd }}$ iteration $\left(f_{1}=64.17 \mathrm{~Hz}\right)$

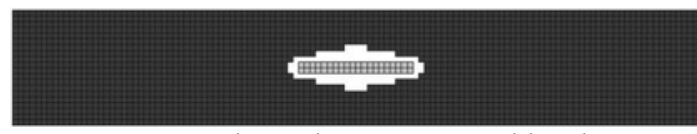

Figure 29: Structural topology generated by the erroneous iteration $\left(f_{1}=0 \mathrm{~Hz}\right)$

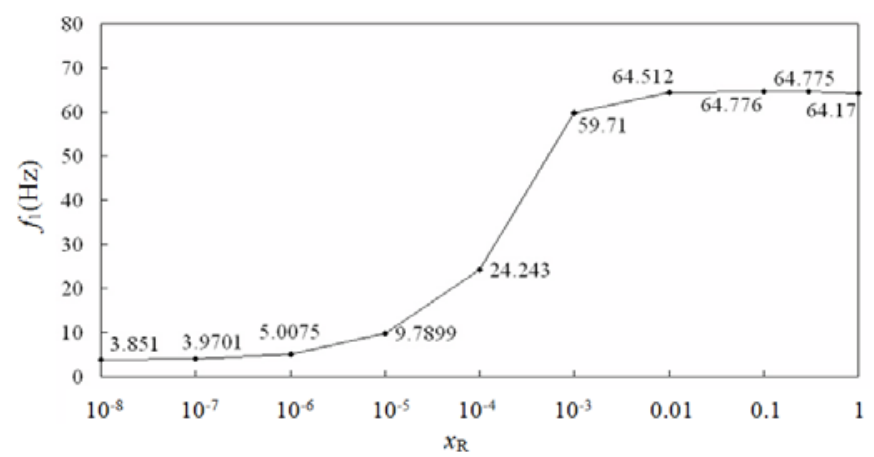

Figure 30: $1^{\text {st }}$ natural frequency $f_{1}$ versus pseudo-density $x_{\mathrm{R}}$

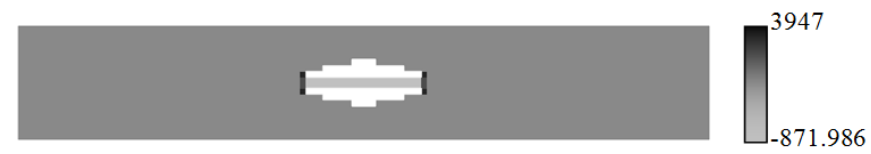

Figure 31: Distribution of sensitivity values with moderate OCM elements $\left(f_{1}=59.193 \mathrm{~Hz}\right)$

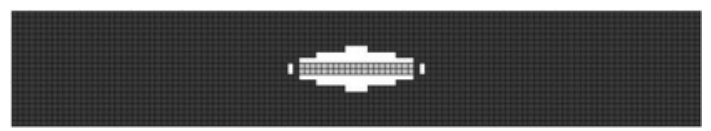

Figure 32 Structural topology generated with CP control $\left(f_{1}=64.273 \mathrm{~Hz}\right)$

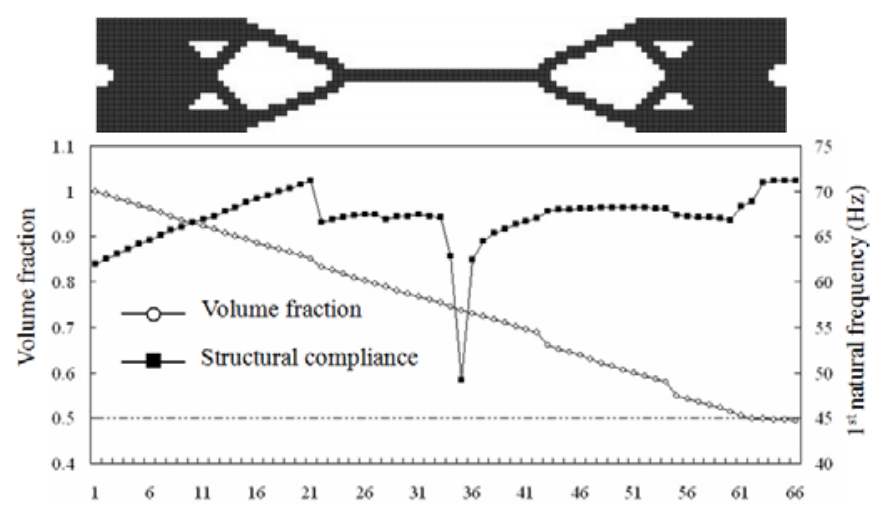

Figure 33: Final optimal design $\left(f_{1}=71.18 \mathrm{~Hz}\right)$ and the iteration history 


\section{Conclusions}

To avoid the erroneous iterations raised in evolutionary optimization procedures, an original CP control method is proposed and integrated with the ERPM method. By introducing the concept of moderate OCM elements, the elements removed erroneously will be replaced in the CP control. These moderate OCM elements will be finally recovered or removed depending upon the direct computing results of sensitivity values. Besides, a threshold method is proposed for a general control of detailed structures and checkerboards that are quantitatively measured by the element bandwidth. Numerical results show that the combination of ERPM, CP control together with the threshold method can deal with both static and dynamic design problems efficiently. The fatal undesired design pattern can be avoided with this new procedure.

\section{Acknowledgements}

This work is supported by the National Natural Science Foundation of China (10676028, 50775184).

\section{References}

1. Xie YM, Steven GP, Shape and layout optimization via an evolutionary procedure. Proc. Int. Conf. Comp. Engrg. Hong Kong 1992

2. Xie YM, Steven GP, Evolutionary Structural Optimization, Springer-Verlag, Berlin, 1997.

3. Xie YM, Steven GP, Optimal design of multiple load case structures using an evolutionary procedure, Engng. Comp. 1994,11: 295-302.

4. Xie YM, Steven GP, A simple approach to structure frequency optimization, Comp. \& Struct. 1994, 53: 14871491.

5. Xie YM, Steven GP, Evolutionary structure optimization for dynamic problems. Comp. \& Struct. 1996, 58: 10671073.

6. Rong JH, Xie YM, An improved method for evolutionary structure optimization against buckling. Comp. \& Struct. 2001, 79: 253-263.

7. Querin OM, Evolutionary structure optimization: stress based formulation and implementation. Ph.D. Thesis, University of Sydney, 1997.

8. Querin OM, Young V, Computational efficiency and validation of bi-directional evolutionary structure optimization. Comp. Meth. Appl. Mech. Engrg. 2000, 189: 559573.

9. Yang XY, Xie YM, Steven GP, Bidirectional evolutionary method for stiffness optimization. AIAA Journal. 1999, 37: 1483-1488.

10. Yang XY, Xie YM, Steven GP, Topology optimization for frequency using an evolutionary method. J. Struct. Engnrg. 1999, 125: 1432-1438

11. Tanskanen $\mathrm{P}$, The evolutionary structural optimization method: theoretical aspects. Comp. Meth. Appl. Mech. Engrg. 2002, 191: 5485-5498.

12. Sigmund O, A 99 line topology optimization code written in MATLAB. Struct. Multidisc. Optim. 2001, 21: 120 127.

13. Zhou M, Rozvany GIN, On the validity of ESO type methods in topology optimization. Struct. Multidisc. Optim. 2001, 21: 80-83.
14. Rozvany GIN, Stress ratio and compliance based methods in topology optimization - a critical review. Struct. Multidisc. Optim. 2001, 21: 109-119.

15. Jog CS, Haber RB, Stability of finite element models for distributed-parameter optimization and topology design. Comp. Meth. Appl. Mech. Engrg. 1996, 130: 203-226.

16. Rodrigues H, Fernandes P, A material based model for topology optimization of thermoelastic structure. Int. J. Numer. Meth. Engrg. 1995, 38: 1951-1965.

17. Sigmund O, Petersson J, Numerical instabilities in topology optimization: A survey on procedures dealing with checkerboards, mesh-dependencies and local minima. Struct. Multidisc. Optim. 1998, 16: 68-75.

18. Haber RB, Jog CS, Bendsøe MP, A new approach to variable-topology shape design using a constraint on perimeter. Struct. Multidisc. Optim. 1996, 11: 1-12.

19. Zhang WH, Duysinx P, Dual approach using a variant perimeter constraint and efficient sub-iteration scheme for topology optimization. Comp. \& Struct. 2003, 81: 2173-2181.

20. Li Q, Steven GP, Xie YM, A simple checkerboard suppression algorithm for evolutionary structure optimization. Struct. Multidisc. Optim 2001, 22: 230-239

21. Yang XY, Xie YM, Perimeter control in the bi-direction evolutionary optimization method. Struct. Multidisc. Optim 2003, 24: 430-440.

22. Zhu JH, Zhang WH, Evolutionary topology optimization of replaceable orthotropic cellular microstructures, Proceedings of International Conference on Mechanical Engineering and Mechanics, Nanjing, China, October 27-29 2005.

23. Pedersen NL, Maximization of eigenvalues using topology optimization, Struct. Multidisc. Optim. 2000, 20: 2-11.

24. Zhu JH, Zhang WH, Qiu KP, Investigation of localized modes in topology optimization of dynamic structures. Acta Aeronautica et Astronautica Sinica, 2006, 27: 619623.

25. Wang D, Friswell MI, Li Y, Maximizing the natural frequency of a beam with an intermediate elastic support. J. Sound \& Vibration 2006, 291: 1229-1238. 